\title{
Procurement monitoring and coordination through Information Technology to overcome drug shortage in the health sector
}

\author{
Dr. H.D.B. Herath \\ Post graduate Institute of Medicine, University of Colombo \\ Dr. K.D.N.P. Ranaweera \\ Post graduate Institute of Medicine, University of Colombo \\ Dr. E.S.S. Rodrigo \\ Postgraduate Institute of Medicine, University of Colombo. Sri lanka. \\ Contact e-mail address: semuthurodrigo@gmail.com \\ eHealth Sri Lanka 2010,1(suppl.1):S17 \\ DOI: http://dx.doi.org/10.4038/sljbmi.v1i0.3554
}

Only the Abstract is available

\begin{abstract}
The drug bill is an area in which savings can be made by timely rational procurements without an impact on patients. In 2009, drug cost was Rupees 14 billion but actual requirement was Rupees 26 billion. Procurement of drugs through open tenders takes at least nine months. All steps in procurement of drugs are carried out according to the procurement plan. Poor coordination between the departments leads to a delay in procurement procedures invariably result in a shortage and local purchase of drugs at higher prices worsening the financial situation. Delays in procurements beyond the bid validity period give opportunity to bidders to increase their quoted prices aggravating the situation. In view of the above issues, it is proposed to establish a Procurement Monitoring and Coordination Unit (PMCU) consisting of representatives from each unit involving a procurement process through Information Technology at the ministry of health to monitor and coordinate procurement of pharmaceuticals. Pro active approach should be the key strategy of proposed unit. Initially PMCU should monitor only procurement of essential drugs, Ministry and cabinet level drugs only. Whenever a tender file is submitted from one unit to another unit, it should be informed to PMCU through internet. Monthly requirements, stock positions at central and regional drugs stores should be linked to PMCU. In addition, list of essential drugs, CAPC and MPC drugs tender list, testing capacity, time and cost for quality testing and consultant data base for technical evaluation of drugs have to be maintained by the PMCU. If any tender file is stagnated at one unit, automatic warning massages can be sent to that unit through proposed software to expedite the procurement process. If this system functions properly, several billions can be saved avoiding local purchases and shortages of drugs in the health sector.
\end{abstract}

Keywords - PMCU, procurement of drugs, delays in procurements 